IJMMS 27:11 (2001) 645-651

PII. S016117120100744X

http://ijmms.hindawi.com

(C) Hindawi Publishing Corp.

\title{
CONVERGENT NETS IN ABELIAN TOPOLOGICAL GROUPS
}

\author{
ROBERT LEDET
}

(Received 30 April 2001)

\begin{abstract}
A net in an abelian group is called a $T$-net if there exists a Hausdorff group topology in which the net converges to 0 . This paper describes a fundamental system for the finest group topology in which the net converges to 0 . The paper uses this description to develop conditions which insure there exists a Hausdorff group topology in which a particular subgroup is dense in a group. Examples given include showing that there are Hausdorff group topologies on $\mathbb{R}^{n}$ in which any particular axis may be dense and Hausdorff group topologies on the torus in which $S^{1}$ is dense.
\end{abstract}

2000 Mathematics Subject Classification. 22A05.

1. Introduction. Let $G$ be an abelian group and $\left\langle x_{\alpha}\right\rangle_{\alpha \in A}$ a net in $G$. If 0 is the identity element in $G$, we can ask what is the finest group topology on $G$ such that $\left\langle x_{\alpha}\right\rangle_{\alpha \in A}$ converges to 0 ? In the terminology of [1], we are placing the topology of a nonconstant net on the subspace $\left(\left\langle x_{\alpha}\right\rangle_{\alpha \in A} \cup\{0\}\right) \subset G$ and finding the associated Graev topology. Ledet and Clark [2] developed a fundamental system approach to defining group topologies in which a sequence $\left\langle a_{n}\right\rangle_{n=1}^{\infty}$ converges to 0 . When there exists a Hausdorff group topology in which $\left\langle a_{n}\right\rangle_{n=1}^{\infty}$ converges to 0, Zelenyuk and Protasov [3] said that $\left\langle a_{n}\right\rangle_{n=1}^{\infty}$ is a $T$-sequence. The purpose of this paper is to extend some of the results of Ledet and Clark to nets and to investigate some applications of these results. We adopt the terminology of Zelenyuk and Protasov in the following.

DEFINITION 1.1. We say that $\left\langle x_{\alpha}\right\rangle_{\alpha \in A}$ is a $T$-net if there exists a Hausdorff group topology on $G$ in which $\left\langle x_{\alpha}\right\rangle_{\alpha \in A}$ converges to 0 .

We will discover how subgroups can be made dense in Hausdorff group topologies and we use this description to show that there are Hausdorff group topologies on $\mathbb{R}^{n}$ in which any particular axis may be dense and Hausdorff group topologies on the torus in which $S^{1}$ is dense.

We will assume as additional hypothesis throughout that $G$ is an abelian group and each sequence is an injective function from the natural numbers into the group $G$. Also the notations $\mathbb{N}, \mathbb{Z}, \mathbb{R}$, and $S^{1}$ will denote the natural numbers, integers, reals, and the circle group, respectively. We use the notation $H<G$ to mean that $H$ is a subgroup of $G$ and when referring to the element of $G / H$ corresponding to the coset $g+H$, we use the notation $\bar{g}$. Finally, we denote the cardinality of a set $X$ by $|X|$.

2. Fundamental systems generated by nets. Since $G$ is abelian, it is possible to define various fundamental systems on a subgroup of $G$ and use them as a fundamental system for the entire group. We will use the elements of the net $\left\langle x_{\alpha}\right\rangle_{\alpha \in A}$ to 
define such a fundamental system for the subgroup generated by $\left\langle x_{\alpha}\right\rangle_{\alpha \in A}$. For each $\alpha_{0} \in A$, define $T\left(\alpha_{0}\right)=\{0\} \cup\left\{x_{\alpha} \mid \alpha>\alpha_{0}\right\} \cup\left\{-x_{\alpha} \mid \alpha>\alpha_{0}\right\}$ where $-x_{\alpha}$ denotes the inverse of $x_{\alpha}$ in $G$, and let $\zeta$ denote the collection of all nondecreasing sequences in $\mathbb{N}$. Then for each $C=\left\langle c_{n}\right\rangle_{n=1}^{\infty} \in \zeta$ and for each increasing countable subset $A^{\prime}=\left\langle a_{n}\right\rangle_{n=1}^{\infty}$ of $A$, we define

$$
U\left(C, A^{\prime}\right)=\left\{g_{1}+g_{2}+\cdots+g_{k} \mid g_{i} \in c_{i} T\left(\alpha_{i}\right) \text { for } i \in\{1,2, \ldots, k\}, k \in \mathbb{N}\right\},
$$

where $c_{i} T\left(\alpha_{i}\right)$ denotes the set of sums of $c_{i}$ addends, each of which lies in $T\left(\alpha_{i}\right)$.

Proposition 2.1. Let $\mathscr{F}=\left\{U\left(C, A^{\prime}\right) \mid C \in \zeta\right.$ and $A^{\prime} \subset A$ is countable and increasing $\}$. Then $\mathscr{F}$ is a fundamental system for the finest group topology on $G$ for which $\left\langle x_{\alpha}\right\rangle_{\alpha \in A}$ converges to 0 .

Proof. Suppose that $U\left(C, A^{\prime}\right)$ and $U\left(D, B^{\prime}\right)$ are elements of $\mathscr{F}$, where $A^{\prime}=\left\langle a_{i}\right\rangle_{i=1}^{\infty}$ and $B^{\prime}=\left\langle b_{i}\right\rangle_{i=1}^{\infty}$. For each $i \in \mathbb{N}$, let $e_{i}=\min \left\{c_{i}, d_{i}\right\}$ and $E=\left\langle e_{i}\right\rangle_{i=1}^{\infty}$. Now note that there exists $\gamma_{1} \in A$ such that $\alpha_{1} \leq \gamma_{1}$ and $\beta_{1} \leq \gamma_{1}$. Then for $n>1$, we can find $\gamma_{n} \in A$ with $\alpha_{n} \leq \gamma_{n}, \beta_{n} \leq \gamma_{n}$, and $\gamma_{n-1} \leq \gamma_{n}$. Thus $\Gamma=\left\langle\gamma_{i}\right\rangle_{i=1}^{\infty}$ is increasing, $U(E, \Gamma) \in \mathscr{F}$ and $U(E, \Gamma) \subset\left\{U\left(C, A^{\prime}\right) \cap U\left(D, B^{\prime}\right)\right\}$.

Now suppose that $x \in U\left(C, A^{\prime}\right)$. Then $x=g_{1}+g_{2}+\cdots+g_{k}$ where $k \in \mathbb{N}$ and $g_{i} \in$ $c_{i} T\left(\alpha_{i}\right)$ for $i \in\{1,2, \ldots, k\}$. Thus, if $C^{\prime}=\left\langle c_{k+1}, c_{k+2}, \ldots\right\rangle$ and $A^{\prime \prime}=\left\langle\alpha_{k+1}, \alpha_{k+2}, \ldots\right\rangle$, then $x+U\left(C^{\prime}, A^{\prime \prime}\right) \subset U\left(C, A^{\prime}\right)$.

Let $U\left(C, A^{\prime}\right) \in \mathscr{F}$. Now either $C$ is eventually constant of cofinal in $\mathbb{N}$. If $m, n \in \mathbb{N}$ and $c_{i}=n$ for all $i \geq m$, let $C^{\prime}=\left\langle c_{i}\right\rangle_{i=m}^{\infty}$ and $A^{\prime}=\left\langle\alpha_{2 i}\right\rangle_{i=m}^{\infty}$. Then $2 U\left(C^{\prime}, A^{\prime \prime}\right) \subset U\left(C, A^{\prime}\right)$. If $C$ is a cofinal sequence in $\mathbb{N}$, we can find a subsequence $\left\langle c_{n_{i}}\right\rangle_{i=1}^{\infty}$ of $C$ which is strictly increasing. For each $i \in \mathbb{N}$, define

$$
c_{i}^{\prime}= \begin{cases}\frac{c_{2 n_{i}}}{2} & \text { if } c_{2 n_{i}} \text { is even, } \\ \frac{c_{2 n_{i}}-1}{2} & \text { if } c_{2 n_{i}} \text { is odd. }\end{cases}
$$

Then $2 U\left(C^{\prime}, A^{\prime \prime}\right) \subset U\left(C, A^{\prime}\right)$.

Finally we note that since $-U\left(C, A^{\prime}\right)=U\left(C, A^{\prime}\right), \mathscr{F}$ is a fundamental system for $G$.

Now let $\tau$ be any group topology on $G$ for which the net $\left\langle x_{\alpha}\right\rangle_{\alpha \in A}$ converges to 0 and let $U \in \tau$ with $0 \in U$. We choose a symmetric open set $W$ contained in $U$ with $0 \in W$ and a sequence of symmetric open sets, say $V_{1}, V_{2}, \ldots$, with $0 \in V_{i}$ for all $i, 2 V_{1} \subset W$, and $(n+1) V_{n} \subset V_{n-1}$ for $n \geq 2$.

For any $k \in \mathbb{N}$ we have that $V_{1}+2 V_{2}+\cdots+k V_{k} \subset W$. Since $\left\langle x_{\alpha}\right\rangle_{\alpha \in A}$ converges to 0 in $\tau$, we can find a tail of the net contained in $V_{i}$ for each $i$. Choose $\alpha_{i} \in A$ so that $T\left(\alpha_{i}\right) \subset V_{i}$ and $\alpha_{i}>\alpha_{j}$ for $1 \leq j \leq i-1$. Then $k T\left(\alpha_{k}\right) \subset k V_{k}$ and so if $A^{\prime}=\left\langle a_{i}\right\rangle_{i=1}^{\infty}$, we have that $U\left(\mathbb{N}, A^{\prime}\right) \subset W$.

The process used by Ledet and Clark [2] to construct group topologies in which a sequence $\left\langle a_{n}\right\rangle_{n=1}^{\infty}$ converges to 0 in $G$ actually defines a group topology on the subgroup generated by $\left\{a_{n}\right\}_{n=1}^{\infty}$. Recalling that a Hausdorff group topology is also completely regular, we see that the topology generated by a sequence can never yield a connected group topology. 
3. Dense subgroups determined by nets. We can describe topologies on $G$ in which a certain subgroup $H$ is dense in terms of nets which intersect each coset of $H$ in a specific way. We say that the set $\left\{g_{\beta}\right\}_{\beta \in B} \subset G$ is a representative collection of $G / H$ if $g_{\beta_{1}}-g_{\beta_{2}} \notin H$ for any $\beta_{1}, \beta_{2} \in B$ with $\beta_{1} \neq \beta_{2}$, and if for each $\bar{g}_{0} \in G / H$, there exists a $\beta_{0} \in B$ with $\pi\left(g_{\beta_{0}}\right)=\bar{g}_{0}$ where $\pi$ is the natural homomorphism. Since $|B|=|G / H|$, we use $G / H$ as the index set for this collection.

DEFINITION 3.1. Let $H$ be a subgroup of $(G, \tau)$ and let $\left\{g_{\beta}\right\}_{\beta \in G / H}$ be a representative collection of $G / H$. If, for each $\bar{g}_{\beta} \in G / H$, there exists a net $P_{\beta}: A \rightarrow g_{\beta}+H$ such that the net $P: A \times G / H \rightarrow G$ defined by $P(\alpha, \beta)=P_{\beta}(\alpha)$ with the ordering $(\alpha, \beta) \geq\left(\alpha^{\prime}, \beta^{\prime}\right)$ if and only if $\alpha \geq \alpha^{\prime}$ is a $T$-net, then we say that $P$ is $\tau$-like.

Proposition 3.2. Let $H<G$. There exists a Hausdorff group topology on $G$ in which $H$ is dense if and only if there is a $\tau$-like net $P: A \times G / H \rightarrow G$.

Proof. Suppose that $H$ is dense in $G$ in some Hausdorff group topology $\tau$ and let $\mathscr{F}$ be a fundamental system for $\tau$. Also let $\left\{g_{\beta}\right\}_{\beta \in G / H}$ be a representative collection of $G / H$ and let $g_{\beta} \in\left\{g_{\beta}\right\}_{\beta \in G / H}$. Note that for each $U \in \mathscr{F}, U \cap\left(g_{\beta}+H\right)$ is nonempty. Thus, we can find a net $P_{\beta}: A \rightarrow g_{\beta}+H$ which converges to 0 in $(G, \tau)$. Define $P: A \times G / H \rightarrow G$ by $P\left(\alpha, g_{\beta}\right)=p_{\beta}(\alpha)$, then clearly $P$ is $\tau$-like.

Now suppose that there exists a $\tau$-like net $P: A \times G / H \rightarrow G$ and let $\mathscr{F}$ be the fundamental system generated by $P$ as described in Proposition 2.1. Let $U \in \mathscr{F}$ and $g \in G$. Then $U \cap(g+H)$ is nonempty since $g \in g_{\beta}+H$ for some $g_{\beta} \in\left\{g_{\beta}\right\}_{\beta \in G / H}$. It follows that $H$ is dense in $G$ with the topology generated by $\mathscr{F}$.

As you would expect, algebra plays a large role in determining whether there is a Hausdorff group topology in which a net converges. For example, we can describe $T$-sequences using factorization in the group. The following extends results found in [3].

DeFinITION 3.3. Let $S \subset G, n \in \mathbb{N}$ and $g \in G$. We say that $g$ has an $n$-factorization in $S$ if and only if there exists a collection of nonzero elements of $S$, say $s_{1}, s_{2}, \ldots, s_{n}$, with $g=s_{1}+s_{2}+\cdots+s_{n}$. Two $n$-factorizations, $s_{1}, s_{2}, \ldots, s_{n}$ and $t_{1}, t_{2}, \ldots, t_{n}$, are disjoint if and only if $s_{i} \neq t_{j}$ whenever $i, j \in\{1,2, \ldots, n\}$.

LEMMA 3.4. Let $\left\langle a_{n}\right\rangle_{n=1}^{\infty}$ be a sequence in $G$. Then $\left\langle a_{n}\right\rangle_{n=1}^{\infty}$ is a T-sequence if and only if for each $g \in G$ and $n_{0} \in \mathbb{N}$, there exist only finitely many pairwise disjoint $n_{0}$-factorizations of $g$ in $\left\{a_{n}\right\}_{n=1}^{\infty} \cup\left\{-a_{n}\right\}_{n=1}^{\infty}$.

Proof. Suppose that for some nonzero $g \in G$ and $n_{0} \in \mathbb{N}$, there exist infinitely many pairwise disjoint $n_{0}$-factorizations of $g$ in $\left\{a_{n}\right\}_{n=1}^{\infty} \cup\left\{-a_{n}\right\}_{n=1}^{\infty}$ and suppose $\left\langle a_{n}\right\rangle_{n=1}^{\infty}$ converges to 0 in $\tau$. Then if $U$ is an open set containing 0 and $V$ is a symmetric open set satisfying $0 \in V$ and $n_{0} V \subset U$, we can find a tail of $\left\langle a_{n}\right\rangle_{n=1}^{\infty}$ contained in $V$, say the $m$-tail. Thus, we can find an $n_{0}$-factorization of $g$ in $\left\{a_{n}\right\}_{n=m}^{\infty} \cup\left\{-a_{n}\right\}_{n=m}^{\infty}$ and $g \in n_{0} V$ and $n_{0} V \subset U$. Since $U$ is arbitrary, $\tau$ is not Hausdorff and $\left\langle a_{n}\right\rangle_{n=1}^{\infty}$ is not a $T$-sequence.

Now suppose that for each $g \in G$ and $n_{0} \in \mathbb{N}$, there exist only finitely many pairwise disjoint $n_{0}$-factorizations of $g$ in $\left\{a_{n}\right\}_{n=1}^{\infty} \cup\left\{-a_{n}\right\}_{n=1}^{\infty}$. Then clearly for each $k \in \mathbb{N}$ we 
can find an $n_{1} \in \mathbb{N}$ with no $k$-factorization of $g$ in $T\left(n_{1}\right)$. Thus if $\left\{n_{i}\right\}_{i=1}^{k}$ is any collection such that $n_{1}<n_{2}<\cdots<n_{k}$, then $g \notin T\left(n_{1}\right)+T\left(n_{2}\right)+\cdots+T\left(n_{k}\right)$. Note that there may exist infinitely many $k$-factorizations of $g$ which are not pairwise disjoint. So suppose that for every $n_{k+1}>n_{k}, g \in T\left(n_{1}\right)+T\left(n_{2}\right)+\cdots+T\left(n_{k}\right)+T\left(n_{k+1}\right)$. Since there are only finitely many pairwise disjoint $(k+1)$-factorizations of $g$ in $\left\{a_{n}\right\}_{n=1}^{\infty} \cup\left\{-a_{n}\right\}_{n=1}^{\infty}$, there must exist some collection $s_{1}, s_{2}, \ldots, s_{k}^{\prime}$, where $k^{\prime} \leq k$, and infinitely many $(k+1)$-factorizations of $g$, say $F_{1}, F_{2}, \ldots$, such that $\left\{s_{1}, s_{2}, \ldots, s_{k}^{\prime}\right\} \subset F_{i}$ for each $i$ and $F_{n} \cap F_{m}=\left\{s_{1}, s_{2}, \ldots, s_{k}^{\prime}\right\}$ whenever $m \neq n$. But then there are infinitely many pairwise disjoint $\left(k-k^{\prime}+1\right)$-factorizations of $g-s_{1}-s_{2}-\cdots-s_{k}^{\prime}$, a contradiction. Repeating this process, we find a sequence $\left\langle n_{i}\right\rangle_{i=1}^{\infty}$ with $g \notin U\left(\mathbb{N},\left\langle n_{i}\right\rangle_{i=1}^{\infty}\right)$.

So we see that if $H$ is an infinite subgroup of finite index in $G$, then we can always find such a net.

Corollary 3.5. Suppose that $H$ is an infinite subgroup of $G$ and $G / H$ is finite. Then there exists a Hausdorff group topology on $G$ in which $H$ is dense.

Proof. It is known that every infinite abelian group admits a nondiscrete metrizable group topology and hence we can find a nontrivial $T$-sequence $\left\langle a_{n}\right\rangle_{n=1}^{\infty}$ contained in $H$. Let $\left\{g_{1}, g_{2}, \ldots, g_{m}\right\}$ be a representative collection of $G / H$ and let $\left\{\left\langle b_{i, n}\right\rangle_{n=1}^{\infty}\right\}_{i=1}^{m}$ be any collection of $m$ disjoint subsequences of $\left\langle a_{n}\right\rangle_{n=1}^{\infty}$. For each $i \in\{1,2, \ldots, m\}$ and for each $n \in \mathbb{N}$, define $x_{i, n}=g_{i}+b_{i, n}$. Then note that for each $i,\left\langle x_{i, n}\right\rangle_{n=1}^{\infty} \subset\left(g_{i}+H\right)$.

Without loss of generality, we may assume that the net

$$
\left\langle x_{i, n} \mid i \in\{1,2, \ldots, m\}, n \in \mathbb{N}\right\rangle
$$

is linearly ordered and hence may be considered to be a sequence. Now pick $g \in$ $G$ and suppose that there are infinitely many pairwise disjoint factorizations of $g$ in $\left\langle x_{i, n} \mid i \in\{1,2, \ldots, m\}, n \in \mathbb{N}\right\rangle$. Since $G / H$ is finite, there must exist some collection $\left\{k_{1}, k_{2}, \ldots, k_{m}^{\prime}\right\} \subset \mathbb{Z}$ such that $\sum_{i=1}^{m}\left|k_{i}\right|=n_{0}$ and infinitely many of the $n_{0^{-}}$ factorizations of $g$ are of the form

$$
k_{1}^{\prime} g_{1}+\sum_{i=1}^{\left|k_{1}\right|} c_{1, i} b_{1, n_{i}}+k_{2}^{\prime} g_{2}+\sum_{i=1}^{\left|k_{2}\right|} c_{2, i} b_{2, n_{i}}+\cdots+k_{m}^{\prime} g_{m}+\sum_{i=1}^{\left|k_{m}\right|} c_{m, i} b_{m, n_{i}},
$$

where $c_{i, j} \in\{-1,1\}$ for each $i, j$ and $\left|k_{i}^{\prime}\right| \leq\left|k_{i}\right|$ for each $i$. But then

$$
\mathfrak{g}-k_{1} g_{1}-k_{2} g_{2}-\cdots-k_{m} g_{m}=\sum_{i=1}^{\left|k_{1}\right|} c_{1, i} b_{1, n_{i}}+\sum_{i=1}^{\left|k_{2}\right|} c_{2, i} b_{2, n_{i}}+\cdots+\sum_{i=1}^{\left|k_{m}\right|} c_{m, i} b_{m, n_{i}}
$$

for each $i$. Since each factorization is pairwise disjoint and the sequences $\left\langle b_{i, n}\right\rangle_{n=1}^{\infty}$ are disjoint, there must be infinitely many pairwise disjoint $\left(\left|k_{1}\right|+\left|k_{2}\right|+\cdots+\left|k_{m}\right|+1\right)$ factorizations of $g-k_{1} g_{1}-k_{2} g_{2}-\cdots-k_{m} g_{m}$ in $\left\{a_{n}\right\}_{n=1}^{\infty} \cup\left\{-a_{n}\right\}_{n=1}^{\infty}$, contradicting the fact that $\left\langle a_{n}\right\rangle_{n=1}^{\infty}$ is a $T$-sequence in $G$. Therefore $\left\langle x_{i, n} \mid i \in\{1,2, \ldots, m\}, n \in \mathbb{N}\right\rangle$ is $T$-like and thus generates a Hausdorff group topology on $G$ in which $H$ is dense.

We would certainly like to extend this result since even the standard examples of groups containing dense subgroups do not satisfy these conditions. We do so by 
generalizing the definition of finite index and determining when there exists a net as described in Proposition 3.2.

DEFINITION 3.6. An $n$-factorization $s_{1}, s_{2}, \ldots, s_{n}$ is favorable if and only if $s_{i} \neq s_{j}$ whenever $i \neq j$.

Definition 3.7. Let $H<G$ and let $\left\langle x_{\alpha}\right\rangle_{\alpha \in A}$ be a net in $H$. Then $\left(G, H,\left\langle x_{\alpha}\right\rangle_{\alpha \in A}\right)$ is compatible if and only if there exists a representative collection $\left\{g_{\beta}\right\}_{\beta \in G / H}$ of $G / H$ such that whenever $g \in G$ with $g=g_{\beta}+h$, there exists an increasing sequence $\left\langle a_{n}\right\rangle_{n=1}^{\infty} \subset A$ such that whenever $h^{\prime} \in H$ and $g+h^{\prime}$ has a favorable $n$-factorization in $\left\{g_{\beta}\right\}_{\beta \in G / H} \cup$ $\left\{-g_{\beta}\right\}_{\beta \in G / H}$, then $h-h^{\prime}$ has no favorable $n$-factorization $s_{1}, s_{2}, \ldots, s_{n}$ such that $s_{i} \in$ $T\left(\alpha_{i}\right)$ for each $i$.

THEOREM 3.8. Let $H<G$ and let $\left\langle x_{\alpha}\right\rangle_{\alpha \in A}$ be a net in $H$. If

(i) $\left(G, H,\left\langle x_{\alpha}\right\rangle_{\alpha \in A}\right)$ is compatible, and

(ii) there exists $|G / H|$ subnets of $\left\langle x_{\alpha}\right\rangle_{\alpha \in A}$ such that any two of the subnets have disjoint tails,

then there exists a Hausdorff group topology on $G$ in which $H$ is dense.

Proof. Without loss of generality, assume that $0 \notin\left\{x_{\alpha}\right\}_{\alpha \in A}$. By condition (ii), for each $\beta \in G / H$, we can find a subnet of $\left\langle x_{\alpha}\right\rangle_{\alpha \in A}$, say $\left\langle x_{\beta, \lambda} \mid \lambda \in A_{\beta}\right\rangle$, where $A_{\beta}$ is a cofinal subset of $A$. Define $P: \cup_{\beta \in G / H}\{\beta\} \times A_{\beta} \rightarrow G$ by $P(\beta, \lambda)=g_{\beta}+x_{\beta, \lambda}$. Recalling $A_{\beta} \subset A$ for each $\beta$, we partially order $\cup_{\beta \in G / H}\{\beta\} \times A_{\beta}$ by $\left(\beta_{1}, \lambda_{1}\right) \leq\left(\beta_{2}, \lambda_{2}\right)$ if and only if $\lambda_{1} \leq_{A} \lambda_{2}$ where $\leq_{A}$ is the relation which directs $A$. We denote the resulting net

$$
\left\langle g_{\beta}+x_{\beta, \lambda} \mid \beta \in G / H, \lambda \in A_{\beta}\right\rangle .
$$

Now let $g \in G$. Then there exists a unique $g_{\beta} \in\left\{g_{\beta}\right\}_{\beta \in G / H}$ and $h \in H$ such that $g=g_{\beta}+h$. Also, by condition (i) there exists an increasing sequence $\left\langle\alpha_{n}\right\rangle_{n=1}^{\infty}$ in $A$ such that whenever $h^{\prime} \in H$ and $g+h^{\prime}$ has a favorable $n_{0}$-factorization in $\left\{g_{\beta}\right\}_{\beta \in G / H} \cup$ $\left\{-g_{\beta}\right\}_{\beta \in G / H}$, then $h-h^{\prime}$ has no favorable $n_{0}$-factorization $s_{1}, s_{2}, \ldots, s_{n}$ such that $s_{i} \in$ $T\left(\alpha_{i}\right)-\{0\}$ for each $i$. For each $\alpha \in A$, let

$$
T^{\prime}(\alpha)=\{0\} \cup\left\{g_{\beta}+x_{\beta, \lambda} \mid \lambda \geq \alpha\right\} \cup\left\{-\left(g_{\beta}+x_{\beta, \lambda}\right) \mid \lambda \geq \alpha\right\}
$$

and suppose for some $n \in \mathbb{N}, g \in T^{\prime}\left(\alpha_{1}\right)+T^{\prime}\left(\alpha_{2}\right)+\cdots+T^{\prime}\left(\alpha_{n}\right)$. But for every favorable $n_{0}$-factorization with $g_{i} \in\{-1,1\}, g_{\beta_{i}}+x_{\beta_{i}, \lambda_{i}} \in T^{\prime}\left(\alpha_{i}\right)$ for each $i$ and $\sum_{i=1}^{n} c_{i} g_{\beta_{i}}=$ $g_{\beta}+h^{\prime}$ for some $h^{\prime} \in H$, we must have that $\sum_{i=1}^{n} c_{i} x_{\beta_{i}, \lambda_{i}}=h-h^{\prime}$ since $\left(G, H,\left\langle x_{\alpha}\right\rangle_{\alpha \in A}\right)$ is compatible. Thus $g \notin T^{\prime}\left(\alpha_{1}\right)+T^{\prime}\left(\alpha_{2}\right)+\cdots+T^{\prime}\left(\alpha_{n}\right)$ for any $n \in \mathbb{N}$ and hence $g \notin U^{\prime}\left(\langle 1\rangle,\left\langle\alpha_{n}\right\rangle_{n=1}^{\infty}\right)$ where $\langle 1\rangle$ denotes the constant 1 sequence and

$$
U^{\prime}\left(\langle 1\rangle,\left\langle\alpha_{n}\right\rangle_{n=1}^{\infty}\right)=\left\{g_{1}+g_{2}+\cdots+g_{k} \mid g_{i} \in T^{\prime}\left(\alpha_{i}\right) \text { for } i \in\{1,2, \ldots, k\}, k \in \mathbb{N}\right\} .
$$

Therefore $\left\langle g_{\beta}+x_{\beta, \lambda}\right\rangle$ is a $T$-net.

By Proposition 3.2, $H$ is dense in the topology generated by

$$
\left\langle g_{\beta}+x_{\beta, \lambda} \mid \beta \in G / H, \lambda \in A_{\beta}\right\rangle \text {. }
$$


In order to give examples of groups satisfying the conditions of Theorem 3.8, we note that there exists uncountably many increasing sequences in $\mathbb{N}$ such that the intersection of any two is finite.

COROLLARY 3.9. There exist Hausdorff group topologies on $\mathbb{R}$ in which $\mathbb{Z}$ is dense.

PRoof. Let $\left\langle\alpha_{n}\right\rangle_{n=1}^{\infty}$ be an increasing sequence in $\mathbb{Z}$ such that $a_{n+1} / a_{n}>n$ for all $n \in \mathbb{N}$ and note that $[0,1)$ is a representative collection of $\mathbb{R} / \mathbb{Z}$. So pick $r \in \mathbb{R}$. Then there exists a unique $c \in[0,1)$ and $k \in \mathbb{Z}$ with $r=c+k$. For each let $i \in \mathbb{N}$, let $n_{i}=$ $|k|+i+1$ and note that $a_{n+1}-n a_{n}>a_{n}$ for every $n$. Also if $m \in \mathbb{N}$ and $\left\{c_{i}\right\}_{i=1}^{m} \subset[0,1)$ with $\sum_{i=1}^{m} c_{i}=c+k^{\prime}$, then $\left|k^{\prime}\right|<m$. But for every subset $\left\{g_{i}\right\}_{i=1}^{m}$ of $\left\{a_{n}\right\}_{n=1}^{\infty} \cup\left\{-a_{n}\right\}_{n=1}^{\infty}$ with $g_{i} \in T\left(a_{n_{i}}\right)$ for each $i$, we have that $\left|\sum_{i=1 s}^{m} g_{i}\right|>a_{n_{m}}-(m-1) a_{n_{m}-1}>a_{n_{m}-1}$. But certainly $a_{n_{m-1}}>|k|+m>\left|k-k^{\prime}\right|$ since $n_{m}-1=|k|+m$. Therefore, $\left(\mathbb{R}, \mathbb{Z},\left\langle\alpha_{n}\right\rangle_{n=1}^{\infty}\right)$ is compatible and by the above, there exists a Hausdorff group topology on $\mathbb{R}$ in which $\mathbb{Z}$ is dense.

Next we prove a result which will supply us with a large number of examples of groups containing dense subgroups.

Proposition 3.10. Suppose that $G, H$ are infinite abelian groups. If there exists $|G|$ linearly independent elements of $H$, then there exists a Hausdorff group topology on $G \times H$ in which $H$ is dense.

Proof. Note that $\{(g, 0) \mid g \in G\}$ is a representative collection of $G \times H / H$ and let $(g, h) \in[(G \times H)-\{(0,0)\}]$. If $h \neq 0$, then we can find an increasing sequence $\left\langle a_{n}\right\rangle_{n=1}^{\infty} \subset A$ such that $h \notin U\left(\langle 1\rangle,\left\langle a_{n}\right\rangle_{n=1}^{\infty}\right)$ since $\left\langle x_{\alpha}\right\rangle_{\alpha \in A}$ is a $T$-net. If $h=0$ and $\left\langle\alpha_{n}\right\rangle_{n=1}^{\infty}$ is any increasing sequence in $A$, then $(g, 0)$ has no favorable factorization in $T\left(\alpha_{1}\right)$ by condition (i). Therefore $\left(G \times H, H,\left\langle\left(0, x_{\alpha}\right)\right\rangle_{\alpha \in A}\right)$ is compatible and there exists a Hausdorff group topology on $G \times H$ in which $H$ is dense.

So for example, we see that if $G$ is an abelian group with $|G| \leq|\mathbb{R}|$. Then there exists a Hausdorff group topology on $G \times \mathbb{R}$ in which $\mathbb{R}$ is dense. If we impose the same cardinality restraints on $G$, then we can similarly show that there exists a Hausdorff group topology on $G \times S^{1}$ in which $S^{1}$ is dense.

Thus there are Hausdorff group topologies on $\mathbb{R}^{n}$, for any $n \in \mathbb{N}$, in which any particular axis may be dense and Hausdorff group topologies on the torus in which $S^{1}$ is dense.

We note that Proposition 3.2 does impose some limitations on which subgroups may be dense in a group and we end this section with a result concerning this. We denote the power set of a set $X$ by $P(X)$.

Proposition 3.11. If $H<G$ and $|G|>2^{|H|}$, then there is no Hausdorff group topology on $G$ in which $H$ is dense.

Proof. If $H$ is dense in $(G, \tau)$, then for each $\bar{g} \in G / H$, there must exist a net $\left\langle x_{g, \alpha}\right\rangle_{\alpha \in A} \subset(g+H)$ such that $\left\langle x_{g, \alpha}\right\rangle_{\alpha \in A}$ converges to 0 in $\tau$. Note that if $g_{1}-g_{2} \notin H$, $\left\langle x_{g_{1}, \alpha}-g_{1}\right\rangle_{\alpha \in A}$ and $\left\langle x_{g_{2}, \alpha}-g_{2}\right\rangle_{\alpha \in A}$ must have distinct tails since $\tau$ is Hausdorff. But $\left\{x_{\mathfrak{g}, \alpha}-g_{0} \mid \bar{g}_{0} \in G / H, \alpha \in A\right\} \subset H$. Clearly this is a contradiction since $|P(H)|<|G|=$ $|G / H|$. 


\section{REFERENCES}

[1] B. Clark and V. Schneider, On constructing the associated Graev topology, Arch. Math. (Basel) 55 (1990), no. 3, 296-297. MR 91m:22003. Zbl 716.22001.

[2] R. Ledet and B. Clark, $T_{\Omega}$-sequences in Abelian groups, Int. J. Math. Math. Sci. 24 (2000), no. 3, 145-148. MR 2001d:22002. Zbl 956.22001.

[3] E. Zelenyuk and I. Protasov, Topologies on abelian groups, Math. USSR Izv. 37 (1991), 445-460, [translated from Izv. Akad. Nauk SSSR Ser. Mat. 54 (1990), 1090-1107. MR 93b:22001]. Zbl 728.22003.

ROBERT LEDET: 200 WINGFIELD DRIVE, HOUMA, LA 70360, USA

E-mail address: r1edet@entergy.com 


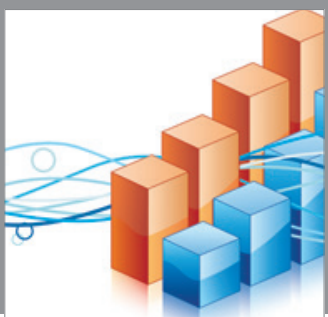

Advances in

Operations Research

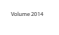

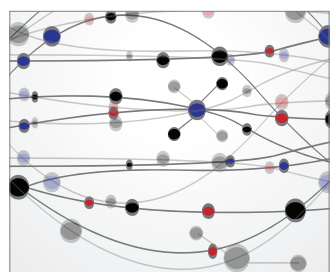

\section{The Scientific} World Journal
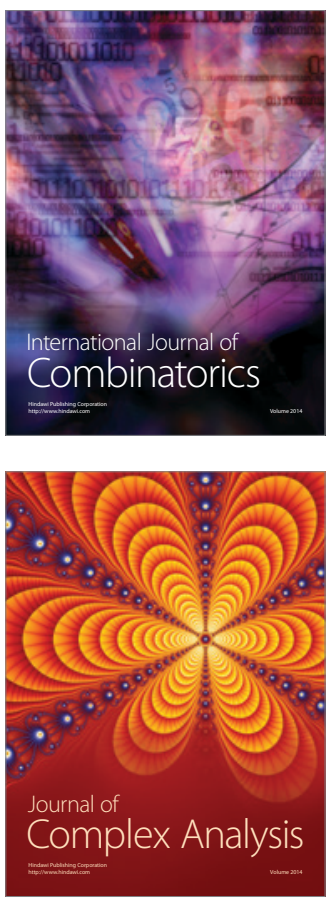

International Journal of

Mathematics and

Mathematical

Sciences
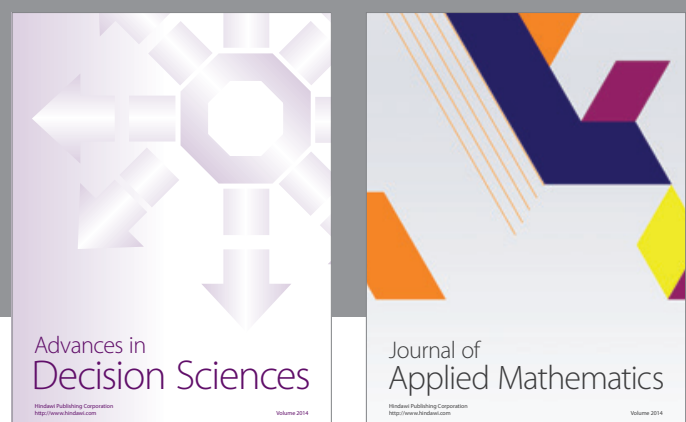

Journal of

Applied Mathematics
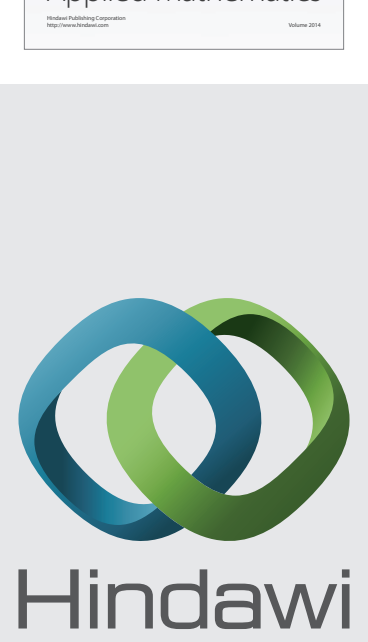

Submit your manuscripts at http://www.hindawi.com
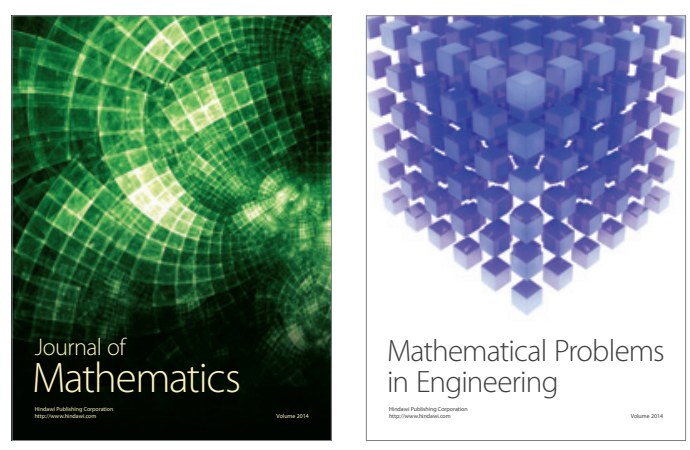

Mathematical Problems in Engineering
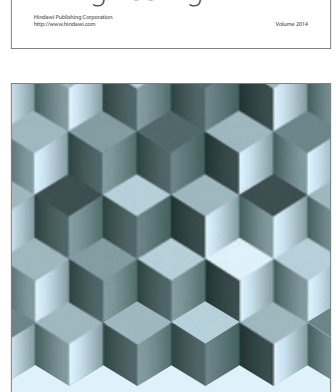

Journal of

Function Spaces
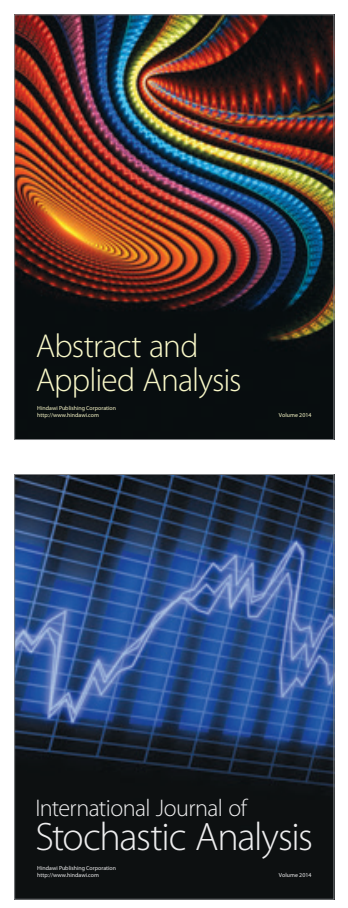

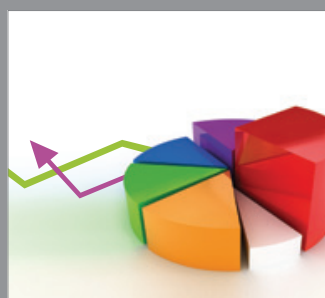

ournal of

Probability and Statistics

Promensencen
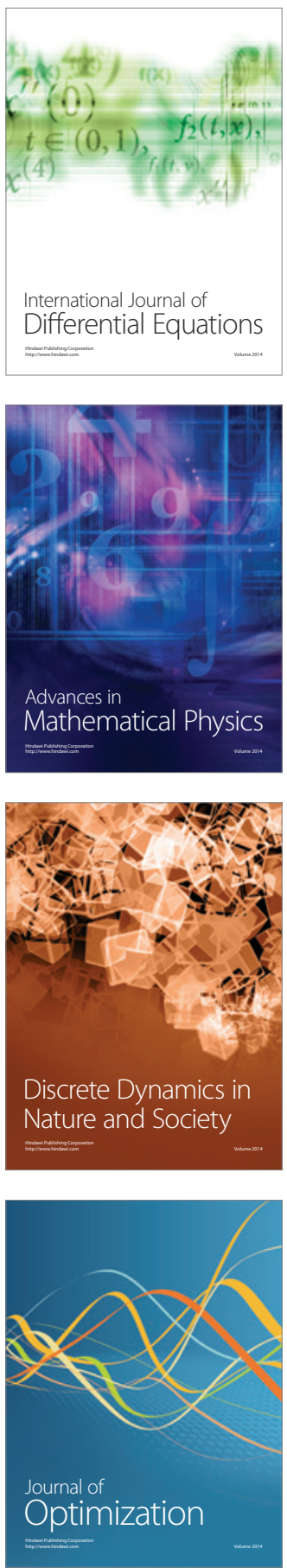